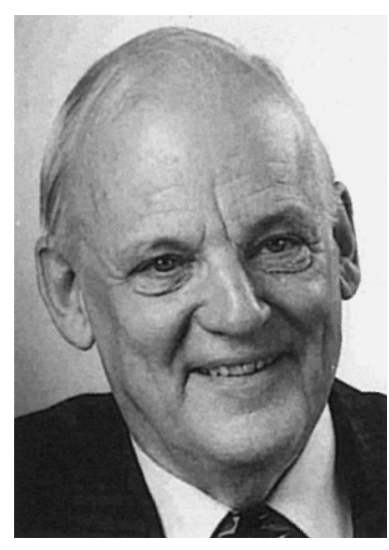

Gösta Samuelson gosta.samuelson@htu.se

\section{Probiotics in health and disease}

$\mathrm{P}$ robiotics are products containing living, non-pathogenic microorganisms, particularly lactobacilli and bifidobacteria, which have beneficial effects. Each strain has different characteristics. Well-controlled human studies are essential before stating any health claims. It is, however, important to distinguish between beneficial effects on health including disease reduction, and use as part of the treatment of a specific disease. In this issue of $\mathrm{SJN}$, a comprehensive special article by Nils-Georg Asp and Torkel Wadström summarizes lectures presented at a conference on probiotics in September 2003 in Stockholm, Sweden. In four additional review articles on the topic "Probiotics in gastric and intestinal disorders" the latest news in this rapidly expanding scientific field is published.

\section{Probiotics in Helicobacter pylori infections}

Several gastrointestinal diseases, e.g. chronic gastritis and peptic ulcer, are caused by $H$. pylori infection. Antibiotics and proton pump inhibitors are nowadays the therapy of choice. Probiotics have been considered and used to complement the treatment of infection in both in vitro and in vivo studies. In a review article in this issue of $\mathrm{SJN}$, Antonio Gasbarrini et al. give an overview of studies using different Lactobacillus species. From in vitro studies, the direct antimicrobial effect of Lactobacillus species is proposed to occur through competition with $H$. pylori, thus explaining the clinical improvement in patients treated with probiotics. However, although the results are promising, probiotics cannot yet be suggested as a valid alternative to standard treatment of $H$. pylori infections.

\section{Probiotics in irritable bowel disease}

The cause of the gastrointestinal condition irritable bowel syndrome (IBS), with symptoms such as abdominal pain and excessive flatulence, is not known. Motility disturbances and psychological mechanisms have been proposed. An interesting finding presented in a review article by Jennifer A. J. Madden is that the intestinal microflora in people with IBS differs from that of healthy individuals. In comparison with healthy people these patients harbour higher numbers of facultative microbes and low numbers of lactobacilli and bifidobacteria. Treatment with probiotics has been used but the results are ambiguous. A preventive approach with lactobacillus may be of more value than when it is used in the treatment of IBS, but so far this has not been proven.

\section{Probiotics and bacterial translocations}

Many studies have shown that after extensive gastrointestinal surgery or liver transplantation, patients who are unsuitable for enteral feeding have a high frequency of septicaemia caused by bacterial species from the intestinal tract. In a review article Bengt Jeppsson et al. describe different ways to decrease bacterial translocation by using probiotics. By altering the luminal bacterial milieu a reduction in postoperative infections seems possible. The authors' own results are promising but need to be confirmed in larger prospective studies.

\section{Probiotics and safety}

The use of probiotics has increased during the past few decades, particularly in dairy products. In an article written by Seppo Salminen et al. the history of the safe use of current probiotics is reviewed. They comment critically on studies on the risk of infections, toxicity, deleterious metabolic activities and antibiotic resistance in relation to each probiotic strain.

In safety assessment, infants and children may be especially sensitive during a period when the intestinal environment and the immune system are under development. However, several studies, even on preterm infants, have not shown any unfavourable effects. Using probiotics has hitherto been safe. 